DOI:

10.1038/nphys868

\title{
Super symmetry
}

The way that spin is woven into the very fabric of the Universe is writ large in the standard model of particle physics. In this model, which took shape in the 1970s and can explain the results of all particlephysics experiments to date, matter (and antimatter) is made of three families of quarks and leptons, which are all fermions, whereas the electromagnetic, strong and weak forces that act on these particles are carried by other particles, such as photons and gluons, which are all bosons.

Despite its success, the standard model is unsatisfactory for a number of reasons. First, although the electromagnetic and weak forces have been unified into a single force, a 'grand unified theory' that brings the strong interaction into the fold remains elusive. Second, the origins of mass are not fully understood. Third, gravity is not included.

Moreover, there are other, less obvious problems with the standard model. The two natural mass scales in nature are zero and the Planck mass, $\sim 10^{19} \mathrm{GeV} \mathrm{c}^{-2}$. Neither photons nor gluons (which carry the electromagnetic and strong forces, respectively) have mass, but the $W$ and $Z$ bosons that are responsible for the weak force have masses of about $90 \mathrm{GeV} \mathrm{c}^{-2}$. Where does this mass scale come from?

This 'hierarchy problem' can be solved by fine-tuning the model so that various quantum fluctuations cancel out, although many physicists are uncomfortable with this solution because some parameters must be fine-tuned to better than 1 part in $10^{15}$. However, a form of symmetry between fermions and bosons called supersymmetry offers a much more elegant solution because the quantum fluctuations caused by bosons are naturally cancelled out by those caused by fermions and vice versa.

Symmetry plays a central role in physics. The fact that the laws of physics are, for instance, symmetric in time (that is, they do not change with time) leads to the conservation of energy. These laws are also symmetric with respect to space, rotation and relative motion. Initially explored in the early 1970 s, supersymmetry is a less obvious kind of symmetry, which, if it exists in nature, would mean that the laws of physics do not change when bosons are replaced by fermions, and fermions are replaced by bosons.

Although it is difficult to explain supersymmetry through analogies to classical physics, its consequences are dramatic - it predicts that every fundamental particle has a superpartner with half a unit of spin less. The electron, for instance, has a spin of a half, so its superpartner (which is known as a selectron) has zero spin. This means that the superpartner of a boson is always a fermion and vice versa.

Supersymmetry also plays a central role in theories that attempt to unify the forces in the standard model with gravity by treating fundamental particles as vibrating strings or membranes in 10-dimensional or 11-dimensional spacetimes. In these theories the gravitational force is carried by a spin-two boson called the

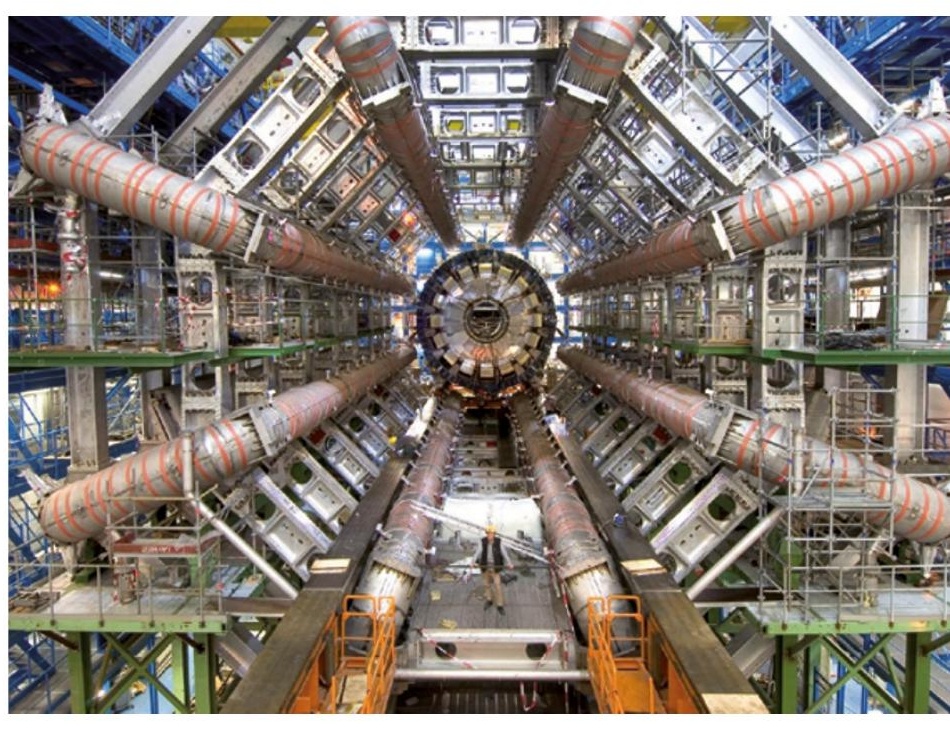

The ATLAS experiment under construction at the Large Hadron Collider. Image courtesy of CERN.

graviton. Searching for supersymmetric particles will be a priority when the Large Hadron Collider comes into operation at CERN, the European particle-physics laboratory near Geneva, in 2008. Peter Rodgers, Chief Editor, Nature Nanotechnology

ORIGINAL RESEARCH PAPERS Golfand, Y. A. $\delta$ Likhtman, E. P. Extension of the algebra of Poincaré group generators and violation of P invariance. JETP Lett. 13, 323-326 (1971) | Neveu, A. \& Schwarz, J. H. Factorizable dual model of pions. Nucl. Phys. B 31, 86-112 (1971) Ramond, P. Dual theory for free fermions. Phys. Rev. D 3, 2415-2418 (1971) | Wess, J. \& Zumino, B. Supergauge transformations in four dimensions. Nucl. Phys. B 70, 39-50 (1974) | Wess, J. \& Zumino, B. A Lagrangian model invariant unde supergauge transformations. Phys. Lett. B 49 , 52-54 (1974)

FURTHER READING Dimopoulos, S., Raby, S. $\&$ Wilczek, F. Supersymmetry and the scale of unification. Phys. Rev. D 24, 1681-1683 (1981)| Almadi, U., de Boar, W. \& Furstenau, H. Comparison of grand unified theories with electroweak and strong coupling constants measured at LEP. Phys. Lett. B 260, 447-455 (1991) | Greene, B. The Elegant Universe (Vintage, London, 2000) I Kane, G. \& Shifman, M. (eds) The Supersymmetric World: The Beginnings of the Theory (World Scientific, Singapore, 2000) 